\title{
Caffeine Duration for Apnea of Prematurity: All Bets Are Off!
}

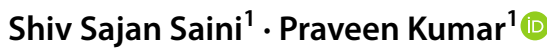 \\ Received: 28 September 2021 / Accepted: 5 October 2021 / Published online: 11 October 2021 \\ (c) Dr. K C Chaudhuri Foundation 2021
}

Caffeine is the most commonly used drug for prevention and treatment of apnea of prematurity and, prevention of bronchopulmonary dysplasia (BPD) [1]. However, several issues with respect to caffeine use in preterm infants are still unresolved. These include optimal gestational cutoff for initiating caffeine, the range of effective dose, and the duration of therapy.

Prakash and colleagues explored optimum duration of therapy by comparing continuation of caffeine till $34 \mathrm{wk}$ postmenstrual age (PMA) vs. stopping caffeine after a $7 \mathrm{~d}$ apnea-free period, in neonates of 26-32 wk gestation [2]. They were willing to accept a $10 \%$ absolute difference in the incidence of 'recurrence of apnea of prematurity (RAP)' against a presumed baseline risk of $1 \%$ among those given caffeine till 34 wk PMA. They randomized a total of 120 neonates against the calculated sample size of 174 neonates for a superiority trial and reported no significant difference in the incidence of RAP between the two groups. The main reason for not being able to find any difference in RAP, if it did exist, lies in the inadequate power of the study. The observed incidence of RAP in the two groups was far higher $(13 \%$ and $15 \%)$ as compared to the presumed baseline risk of $1 \%$ used for sample size estimation, and the observed effect size (2\%) was lower than the presumed effect size of $10 \%$.

With the goal of decreasing exposure to caffeine, an ideal study design to answer this research question should be a noninferiority trial, in which the investigators seek noninferiority of a shorter course of caffeine e.g., 7-d apneafree period compared to caffeine given till 34 wk PMA. The study participants need to be chosen carefully. In the index study, the incidence of RAP in 26-29 wk subgroup was very high-29\% vs. $24 \%$, making it a challenge to stop caffeine early in this subgroup. The incidence of apnea after the infants were otherwise ready for discharge, in the

Praveen Kumar

drpkumarpgi@gmail.com

1 Division of Neonatology, Department of Pediatrics, Post Graduate Institute of Medical Education and Research, Chandigarh 160012, India epidemiological data from Kaiser Permanente Medical Care Program hospitals, ranged from $22.8 \%$ in $29-30$ wk neonates to $26.6 \%$ in $27-28$ wk neonates [3]. Hence, the potential target group may be neonates $>30$ wk gestation in whom the incidence of RAP was $3 \%$ and $6 \%$ in the two groups. A low noninferiority margin is required for such a study as apnea can be a life-threatening event, especially in resourceconstraint settings. As an example, 1794 subjects will be required for a noninferiority trial assuming $96 \%$ success rate of stopping caffeine at $34 \mathrm{wk}$ PMA, with a noninferiority margin of $3 \%$, type 1 error of $2.5 \%$, and power of $90 \%$. A trial aimed at reducing the duration of therapy will also have to measure any changes in the incidence of BPD and neurodevelopmental outcomes, which are potentially benefitted by caffeine. Therefore, while the results of the current study do not inform clinical practice, they surely form the basis for future trials to solve this clinical dilemma.

\section{Declarations}

Conflict of Interest None.

\section{References}

1. Schmidt B, Roberts RS, Davis P, et al; Caffeine for Apnea of Prematurity Trial Group. Caffeine therapy for apnea of prematurity. N Engl J Med. 2006; 354:2112-21.

2. Prakash R, Pournami F, Prabhakar J, Nandakumar A, Nair PMC, Jain N. Duration of caffeine for apnea of prematurity-a randomized controlled trial. Indian J Pediatr. 2021. https://doi.org/10. 1007/s12098-021-03659-y.

3. Lorch SA, Srinivasan L, Escobar GJ. Epidemiology of apnea and bradycardia resolution in premature infants. Pediatrics. 2011;128:e366-73.

Publisher's Note Springer Nature remains neutral with regard to jurisdictional claims in published maps and institutional affiliations. 